\title{
Transfusion transmitted infections among voluntary blood donors from rural areas in coastal Karnataka, India
}

\begin{abstract}
Background: Transfusion of blood and blood products is a lifesaving intervention and benefits numerous patients worldwide. With every unit of blood, there is $1 \%$ chance of transfusion related complications including Transfusion Transmissible Infections (TTIs). This study was undertaken to find out the prevalence of TTI's among voluntary blood donors from rural areas of coastal karnataka, India.
\end{abstract}

Materials and methods: Sera samples from 500 blood donors were screened for Hepatitis B surface antigen, Human Immunodeficiency Virus, Hepatitis C Virus, Syphilis and Malaria. The reactive samples for HIV, HBsAg and HCV were confirmed using a second enzyme-linked immunosorbent assays. Antibodies to Treponema pallidum were confirmed with a Treponema pallidum haemagglutination test (TPHA).

Results: Out of 500 blood donors in the months of April and May 2015, highest number of donors, 249 (49.8\%) belonged to the age group of 21-30 years and the lowest number of donors, $7(1.4 \%)$ belonged to the age group of $51-55$ years. $485(97.0 \%)$ were male donors and $15(3.0 \%)$ were female donors. A maximum number of the blood donors, $219(43.8 \%)$ belonged to $\mathrm{O}$ blood group, followed by blood group A which contained $135(27.0 \%)$ donors. 120 (24.0\%) donors were of B blood group and only 26 (5.2\%) donors belonged to $\mathrm{AB}$ blood group. Of the total donors, $2(0.4 \%)$ of them were positive for HBsAg. Both of them belonged to the age group 21-30 years $(0.8 \%)$ and both of them were males $(0.41 \%)$. One of them was of O blood group $(0.45 \%)$ and the other was of B blood group $(0.83 \%)$.

Conclusion: HBV was the only threat to blood safety in the present study population. Strict selection and retention of voluntary low-risk blood donors is recommended to improve the blood safety.

Keywords: transfusion transmitted infections, hepatitis B, HIV, HBsAg, HCV

\author{
Volume 6 Issue 4 - 2018
}

\author{
Madhushree Barla, I Kirana Pailoor, \\ Murali Keshava S, ${ }^{3}$ Jayaprakash CS, ${ }^{4}$ Olivia \\ D'Cunha, ${ }^{5}$ Lakshmi $C^{6}$ \\ 'Father Muller Medical College, India \\ ${ }^{2}$ Department of Pathology, Father Muller Medical College, India \\ ${ }^{3}$ Department of Paediatrics, Kasturba Medical College, India \\ ${ }^{4}$ Department of Pathology, Father Muller Medical College, India \\ ${ }^{5}$ Technical Supervisor, Father Muller Medical College Hospital, \\ India \\ ${ }^{6}$ Senior Technician, Father Muller Medical College Hospital, India
}

Correspondence: Kirana Pailoor,Associate Professor and Incharge Blood Bank, Department of Pathology, Father Muller Medical College, Kankanady, Mangaluru -575002, India, Tel 9448953716,Email dockirana@yahoo.co.uk

Received: February 22, 2018 | Published: July 03, 2018

\section{Introduction}

Blood is one of the integral components of body constituents, which flows throughout body and becomes a reason for survival. Transfusion of blood and blood products is a lifesaving intervention and benefits numerous patients worldwide. But simultaneously, it carries the risk of transmitting the life- threatening infectious agents. ${ }^{1}$ With every unit of blood, there is $1 \%$ chance of transfusion related complications including Transfusion Transmissible Diseases (TTD). ${ }^{2}$ A majority of known cases of post-transfusion diseases have been caused by Human Immunodeficiency Virus (HIV), Hepatitis B Virus (HBV), Hepatitis C Virus (HCV), Treponima pallidum, Toxoplasma and Malarial parasites. They are the greatest threats to blood safety for transfusion recipients and pose a serious public health problem. Morbidity and mortality resulting from the transfusion of infected blood have far reaching consequences not only for the recipients themselves, but also for their families, communities and the wider society. ${ }^{3}$

Keeping in mind the grave consequences of these infections, to restrain the transmission to minimum, it is very important to remain vigilant about the possible spread of these infections. ${ }^{4}$ Thus screening for Transfusion transmitted infections (TTI's) is essential for blood transfusion safely and in extension for protecting human life. ${ }^{3}$
Basically the aim of the study was to find out the prevalence of TTI's among voluntary blood donors from rural areas in Coastal Karnataka, India.

\section{Material and methods}

This prospective study was done at Father Muller Medical College Hospital Blood Bank. Blood was collected from voluntary blood donors at blood donation camps organized in rural areas at a distance of 50kilometres around Mangalore. This study took into consideration the various TTI's such as HIV, HBV, HCV, Syphilis, Malaria. Study population included voluntary blood donors between the age group ranging between 18 to 55 years. The total sample size was 500 consisting of both male and female blood donors. This study was conducted from April 2015 to May 2015 for a period of 2 months.

A questionnaire consisting of the details such as name, age, sex, date of birth, address and unique ID no. was given to all the donors. Donors with the history of any condition mentioned in the exclusion criteria below were excluded. Detailed history of immunization was noted. Weight, pulse, Blood pressure and temperature were recorded for each donor. Screening for anaemia and inspection for any marks of drug abuse or any skin lesion was recorded. Proper sterilization and other precautions were taken during blood collection and blood units were stored by appropriate methods. 
Patients beyond the range of age consideration and those with the history of hypertension, diabetes mellitus, pre-existing illness in the recent past, uncontrolled weight loss, malaria, recent donation of blood, receipt of blood, who have undergone surgeries and on medications were excluded.

All samples were screened for Hepatitis B surface antigen (Hepalisa $3^{\text {rd }}$ generation-J Mitra \& Co.), Human Immunodeficiency Virus (ELISA, HIV Microlisa 4th generation-J Mitra \& Co.), Hepatitis C Virus (HCV Microlisa $3^{\text {rd }}$ generation-J Mitra \& Co.), Syphilis (Benesphera - Avantor Performance Materials India Limited.) and Malaria (Pan Malaria Card Test- J Mitra\& Co.). Enzyme Linked Immunosorbent Assay method was used to screen and check for seropositivity for HIV, Hepatitis B and Hepatitis C viral infections. Rapid Plasma Reagin method was used to check for the reactivity for Syphilis. Screening for Malarial parasitic infection was done using Rapid card test method. Collected data was analysed by frequency and percentage. Consent was obtained from donors while taking history and ethical clearance was received from the institutional Ethical Committee.

\section{Results}

A total of 500 donors donated blood in the blood donation camp conducted in a period of two months. A maximum number of donors, $249(49.8 \%)$ belonged to the age group of 21-30 years and the least number of donors, $7(1.4 \%)$ belonged to the age group of 51-55 years (Table 1).

Table I Age distribution of the blood donors

\begin{tabular}{lll}
\hline Age (years) & No. of donors & Percentage (\%) \\
\hline $18-20$ & 93 & 18.6 \\
$21-30$ & 249 & 49.8 \\
$31-40$ & 110 & 22 \\
$41-50$ & 41 & 8.2 \\
$51-55$ & 7 & 1.4 \\
Total & 500 & 100 \\
\hline
\end{tabular}

Out of 500 donors, 485 (97.0\%) were males and 15 (3.0\%) were females.

A highest number of blood donors, 219 (43.8\%) belonged to $\mathrm{O}$ blood group, followed by blood group A which contained 135(27.0\%) donors. $120(24.0 \%)$ donors were of B blood group and only 26 (5.2\%) donors belonged to $\mathrm{AB}$ blood group. Of the total 500 donors, 2 were found to be positive for TTIs. Therefore, prevalence of TTIs was $0.4 \%$ (Table 2 ).

Table 2 Occurrence of TTIs among the blood donors

\begin{tabular}{lll}
\hline TTI & No. of subjects positive & Prevalence percentage (\%) \\
\hline HIV & 0 & 0 \\
HBV & 2 & 0.4 \\
HCV & 0 & 0 \\
Syphilis & 0 & 0 \\
Malaria & 0 & 0 \\
Total & 2 & 0.4 \\
\hline
\end{tabular}

Of the total donors, $2(0.4 \%)$ of them were positive for HBsAg. Both of them belonged to the age group 21-30years $(0.8 \%)$ and both of them were males $(0.41 \%)$. One of them was of $\mathrm{O}$ blood group $(0.45 \%)$ and the other was of B blood group $(0.83 \%)$.

\section{Discussion}

Blood is a scarce and life-saving resource; however blood transfusion can be a source for transmitting life threatening infections if screening is not carried out properly. An integrated strategy for Blood Safety is required for elimination of transfusion transmitted infections (TTIs) and for provision of safe and adequate blood. ${ }^{5}$

Out of a total of 500 donors, $485(97.0 \%)$ were males and 15 $(3 \%)$ were females. Fernandes et al., ${ }^{4}$ from Mangalore showed lowest participation by females $(2.5 \%)$ as compared to our study. Nkrumah et al., ${ }^{6}$ showed highest participation by females i.e. $7.8 \%$ indicating the awareness level and fitness level in females in Ghana is more as compared to Indian population. The preponderance of males over females may be due to the fact that Indian females are mostly anaemic and medically unfit for blood donation. Besides low turnout of the females for blood donation may be viewed in the light of comparatively low educational status and the general trend of not involving the female members by the heads of families in such activities. There is a need to create awareness among females and motivating them to come forward to donate blood voluntarily.

Age-wise distribution of the blood donors indicated enthusiastic young age group (21-30 years) contributing maximum, $49.8 \%$ and the least by donors who belonged to the age group of 51-55 years of age which contributed $1.4 \%$. Similarly, in a study conducted by Ampofo et al., ${ }^{7}$ at Ghana, the 21-25 years age group, which included $212(26.2 \%)$ of the donors, was the largest, followed by the 26-30 years age group, with 186 donors $(23.0 \%)$ and the 51-55 years age group included 9 (1.1\%) donors which is less compared to our study. Ray Karmakar et al., ${ }^{8}$ showed that in Kolkata, nearly $68.77 \%$ donors were in the age group of 21- 40 years.

In the present study, the overall rate of seropositivity of HIV was $0.0 \%$. No donor was found to be positive for HIV. Similar result was seen in the study conducted by El- Hazmiet al. ${ }^{9}$ Highest seroprevalence was reported by Ahmed et al., ${ }^{10}$ at Bangladesh (11.7\%) which is in contrast to our study.

As per Government of India Drugs and Cosmetics Act (1945) each blood unit has to be tested for hepatitis B infection, which is mandatory. ${ }^{11}$ According to the WHO classification, this coastal part of Karnataka qualifies as a low prevalence area (less than $2 \%$ ). ${ }^{12}$ In the present study, overall rate of seropositivity of HBsAg was $0.4 \%$. The highest seroprevalence was reported by Ahmed et al. ${ }^{10}$ at Bangladesh $(25 \%)$ and the lowest by Shriyan et al., ${ }^{13}(0.10 \%)$ from Mangalore. The awareness about the disease and modes of prevention may be one reason for the low prevalence and declining trend in HBV infection. Secondly, the implementation of strict pre- donation counseling and donor selection criteria helps in excluding the possibly infected donors. Efforts to control this infection need to be strengthened for the complete prevention of the disease.

In the present study, anti HCV seropositivity among 500 blood donors was $0.0 \%$. No donor was found to be positive for anti $\mathrm{HCV}$. Highest seroprevalence (6\%) was reported by Buseri FI et al, ${ }^{3} 2009$ from Nigeria. The problem with HCV infection may be greater than generally recognized. While effective vaccines currently exist for $\mathrm{HBV}$, a fully protective $\mathrm{HCV}$ vaccine is yet to be available. 
The present study showed that all donors were negative for malaria. The result was similar to the study at Mangalore by Shriyan et al. ${ }^{13}$ Even though the present study showed no prevalence of malaria, it is a high risk condition as Mangalore is endemic to malaria. Thus, screening for malarial parasites is essential. The prevalence rate of syphilis is found to be $0.0 \%$ in the present study. No donor was positive for VDRL. Highest prevalence was reported in Ghana $(13.5 \%)$ in a study by Ampofo et al. ${ }^{7}$

Availability of safe blood for transfusion is a must for the recipients and the community as well. This can be achieved by vigorous screening of donors and donated blood. Effective control strategies including a sensitive and stringent screening of all blood donors, public awareness programs, and institution of adequate public health measures are urgently needed. It may be possible through proper donor selection and education, uniform implementation of laboratory screening tests, and adequate supply of blood through voluntary blood donation along with restriction of donation by professional donors.

\section{Conclusion}

Millions of lives worldwide each year is saved by blood transfusion. But the awareness of both donors and recipients of risk associated with transfusion is less. Accessibility of safe blood for transfusion is an essential for the recipients and the community. To achieve this, screening of donors and donated blood is necessary. So, prevalence of HBV, HCV, and HIV among the blood donors reveals the disease prevalence in the community. Furthermore it estimates the risk of accidental acquisition of these infections during blood transfusion. Motivation and recruitment of potential local blood donor population would help in effective implementing of voluntary blood donation program in the community.

\section{Acknowledgement}

None.

\section{Conflict of interest}

The author declares that there is no conflict of interest.

\section{References}

1. Lathamani K, Bhaktha G, Nayak S, et al. Prevalence of HIV, HCV, HBV and Syphilis in blood donors among the Dakshina Kannada district, India. Int J Curr Microbiol App Sci. 2013;2(10):249-252.
2. Shariff MH, Saldanha P. Seroprevalence of Transfusion Transmitted Infections in a Teaching Hospital Blood Bank. $J$ Evolution Med Dent Sci. 2014;3(6):1459-1462.

3. Buseri FI, Muhibi MA, Jeremiah ZA. Sero-epidemiology of transfusion transmissible infectious diseases among blood donors in Osogbo, southwest Nigeria. Blood Transfus. 2009;7(4):293-299.

4. Fernandes H, D'souza PF, D'souza PM. Prevalence of Transfusion Transmitted Infections in Voluntary and Replacement Donors. Indian $J$ Hematol Blood Transfus. 2010;26(3):89-91.

5. Patel SV, Popat CN, Mazumdar VS, et al. Seroprevalence of HIV, HBV, $\mathrm{HCV}$ and Syphilis in blood donors at a tertiary hospital (Blood Bank) in Vadodara. Int J Med Sci Public Health. 2013;2(3):747-750.

6. Nkrumah B, Owusu M, Frempong HO, et al. Hepatitis B and C viral infections among blood donors from rural Ghana. Ghana Med J. 2011 Sep;45(3):97-100.

7. Ampofo W, Nii-Trebi N, Ansah J, et al. Prevalence of blood- borne infectious diseases in blood donors in Ghana. $J$ Clin Microbiol. 2002;40(9):3523-3525.

8. Karmakar PR, Shrivastava P, Ray TG. Seroprevalence of transfusion transmissible infections at a blood bank of a medical college hospital of Kolkata. Indian J Public Health. 2014;58(1):61-64.

9. El-Hazmi MM. Prevalence of HBV, HCV, HIV-1, 2 and HTLV- I/ II infection among blood donors in a teaching hospital in the Central region of Saudi Arabia. Saudi Med J. 2004;25(1):26-33.

10. Ahmed MV, Begum HA, Hossain T. Incidence of common transfusion transmitted diseases among blood donors. JAFMC Bangladesh. 2009;5(1):04-06.

11. Ghosh A, Singh V, Chawla S. Seroprevalence of Hepatitis B virus infection among the blood donors in a tertiary care hospital Western UP. Sch J App Med Sci. 2015;3(3D):1337-1342.

12. Singh K, Bhat $S$, Shastry $S$. Trend in seroprevalence of Hepatitis B virus infection among blood donors of coastal Karnataka, India. J Infect Dev Ctries. 2009;3(5):376-379.

13. Shriyan AR, Pallipady A, Gomes M. Prevalence of transfusion transmissible infections among blood donors in a tertiary healthcare centre: A five year study. Int J AJ Inst Med Sci. 2012;1(1):32-35. 